\title{
SEEING MORE IS SEEING BETTER
}

Maluf-Filho F. Seeing more is seeing better. Arq Gastroenterol. 2011;48(3):165-6.

HEADINGS - Gastroesophageal reflux, diagnosis. Endoscopy. Diagnosis, computer-assisted.

Non-erosive gastroesophageal reflux disease (NERD) constitutes a formidable diagnostic challenge to the gastroenterologist. The endoscopic examination of the upper gastrointestinal tract fails to detect mucosal breaks of the esophageal lining in up to $40 \%$ of gastroesophageal reflux disease (GERD) patients. Facing this situation it is rather common to rely the diagnosis of GERD on other tests such as $\mathrm{pH}$-monitoring or, more recently, impedance $\mathrm{pH}$-monitoring. Most surprising is the fact that an improvement of heartburn after a trial of proton pump inhibitor (PPI) is highly predictive of the diagnosis of GERD. Those facts point to the fact that a gold standard diagnostic test for GERD is lacking.

Taking these concepts into account we must welcome all the efforts towards the betterment of tests for the diagnosis of GERD. In this issue of the ARQUIVOS de GASTROENTEROLOGIA, Gomes Jr. et al. ${ }^{(1)}$ describe the capability of one method of virtual chromoendoscopy - FICE (Fuji Intelligent Collor Enhancement) - to detect minimal endoscopic lesions (MEL) in patients referred to upper gastrointestinal endoscopy for different reasons including heartburn. Patients with obvious findings of reflux esophagitis, moniliasis, varices and esophagectomy were excluded from the trial. From 224 patients, 155 were sequentially submitted to conventional endoscopic examination of the esophagus and to examination of esophageal mucosa under FICE by the same examiner. Under conventional white light endoscopy, the authors found esophageal MEL in 42 patients (27\%). With FICE, all the 42 patients with esophageal MEL were also detected and 9 patients were also found to have MEL, increasing from $27 \%$ to $33 \%$ the rate of patients with esophageal MEL.

The present results add to the concept that virtual chromoendoscopy enhances our ability to detect mucosal lesions from the pharynx to the rectum. We had the opportunity to test another method of virtual chromoendoscopy (NBI - narrow banding imaging - Olympus Co., Japan) for the detection of superficial esophageal squamous cell cancers in patients with head and neck cancer ${ }^{(2)}$. Comparing NBI to Lugol's chromoendoscopy and white light endoscopy, both NBI and Lugol detected all the cases of superficial neoplasms proving to be superior to conventional white light endoscopy. The main advantage of chromoendoscopy techniques is the fact that they are patient and examiner friendly technologies. Images are automatically obtained pushing a button precluding inherent risks of conventional chromoendoscopy such as hipersensitivity reactions. On the other hand, costs and reproducibility are the main shortcomings of virtual chromoendoscopy. As in any new technique or technology the length of the learning curve and the reproducibility of the results must be carefully evaluated before their incorporation in daily practice. In the article by Gomes Jr. et al..$^{(1)}$, two examiners presented lower performances in detecting esophageal MEL lesion with white light and FICE when compared to the remaining three examiners. The examiner's previous experience and training with the FICE technology is unknown and might have influenced the results. These findings corroborate the intuitive perception that description of virtual chromoendoscopy findings should be standardized in larger forums.

In the article by Gomes Jr. et al. ${ }^{(1)}$, the presence of esophageal MEL lesions did not predict the presence of reflux symptoms. This absence of correlation happened also with the presence of MEL lesions and age (under or over 40), the use of NSAIDs, alcohol and tobacco abuse, and use of PPI. These findings are conflicting with other evidences in the literature. A landmark study by Sharma et al. ${ }^{(3)}$ evaluated narrow band imaging in patients with erosive esophagitis, NERD and in a control population. Narrow band imaging in conjunction with zoom magnification revealed several unique findings not previously described in the NERD patients compared to the controls, including increased number, tortuosity and dilation of intrapapillary capillary vessels, microerosions, and increased vascularity at the squamocolumnar junction. It is a matter of debate whether the use of virtual chromoendoscopy and magnification in the study by Sharma and collaborators could explain the discrepancy in the findings of the studies. Another possible explanation is patient selection. In the investigation conducted by Sharma et al. ${ }^{(3)}$, the definition of patients with and without GERD symptoms was based on two validated GERD questionnaires. So it is possible that the use of validated questionnaires made possible to select patients with the highest and lowest probabilities to have GERD.

Finally, we must pay careful attention to the clinical relevance of the abnormalities found by new 
image technology. Cross sectional image technology such as magnetic resonance imaging and computed tomography-scan offer excellent examples of situations when clinical reasoning is critical to prevent further tests or unnecessary treatments in patients with incidental findings. In the case of NERD patients, the disappearance of esophageal MEL lesions (detected by virtual chromoendoscopy) upon PPI treatment might offer a conclusive proof that seeing more is seeing better.

Fauze MALUF-FILHO*

Maluf-Filho F. Ver mais é ver melhor. Arq Gastroenterol. 2011;48(3):165-6.

DESCRITORES - Refluxo gastroesofágico, diagnóstico. Endoscopia. Diagnóstico por computador.

\section{REFERENCES}

1. Gomes Jr CAR, Loução TS, Carpi G, Catapani WR. A study on the diagnosis of minimal endoscopic lesions in nonerosive reflux esophagitis using computed virtual chromoendoscopy (FICE). Arq Gastroenterol. 2011;48(3): 167-70.
2. Ide E, Maluf-Filho F, Chaves DM, Matuguma SE, Sakai P. NBI assessment for detecting early esophageal squamous cell carcinoma in patients with head and neck cancer: comparative study with lugol staining. World J Gastroenterol. Forthcoming 2011.

3. Sharma P, Wani S, Bansal A, Hall S, Puli S, Mathur S, Rastogi A. A feasibility trial of narrow band imaging endoscopy in patients with gastroesophageal reflux disease. Gastroenterology. 2007;133:454-64. 